\title{
RECONFIRMACIÓN DE GALBA CUBENSIS \\ (GASTROPODA: LYMNAEIDAE), HOSPEDERA DE FASCIOLA HEPATICA (TREMATODA: DIGENEA), POR TÉCNICAS MOLECULARES EN REPÚBLICA DOMINICANA
}

\section{Reconfirmation of Galba cubensis (Gastropoda: Lymnaeidae), host of Fasciola hepatica (Trematoda: Digenea), by molecular techniques in the Dominican Republic}

\author{
Mercedes De Vargas Castro 1a,2a,*, Juan Tomás Camejo Jiménez ${ }^{3}$, Jairo Martínez La Hoz ${ }^{2 b}$, \\ Jean C. Castillo ${ }^{1 b}$, Dolores Paulino ${ }^{1 \mathrm{c}}$ y Pedro $\mathrm{M}^{\mathrm{a}}$. Alarcón-Elbal ${ }^{4}$
}

\begin{abstract}
${ }^{1}$ Laboratorio de investigación de Fasciola hepatica, Instituto Tecnológico de Santo Domingo (INTEC); 1a (D) orcid.org/0000-0002-9726-2079; 1b (D) orcid.org/0000-0001-9290-2412; ic (D) orcid.org/0000-0003-2120-2771. ${ }^{2}$ Universidad Autónoma de Santo Domingo (UASD); 2a,* Para correspondencia: mercedesvargasc24@gmail.com; 2b (D) orcid.org/0000-0001-7249-997X. 3Instituto de Investigaciones Agropecuarias y Forestales (IDIAF), (Dorcid.org/0000-0002-1325-9498 ${ }^{4}$ Instituto de Medicina Tropical \& Salud Global (IMTSAG), Universidad Iberoamericana (UNIBE), Santo Domingo, República Dominica; (D) orcid.org/0000-0001-5319-4257.
\end{abstract}

\section{RESUMEN}

Entre los gasterópodos dulceacuícolas, muchas de las especies de la familia Lymnaeidae están involucradas en la transmisión del trematodo parásito Fasciola hepatica (Linnaeus, 1758) a nivel mundial. Galba cubensis (Pfeiffer, 1839) ha sido señalada como vector en Sudamérica y otras áreas del Neotrópico. El uso de técnicas moleculares ha sido empleado por diferentes grupos de investigadores para discriminar entre especies conquiológicamente indistinguibles, como son las del grupo Galba/Fossaria, esclareciendo aspectos epidemiológicos de crucial importancia en el estudio y control de la fascioliasis. El objetivo de esta investigación fue reconfirmar con técnicas moleculares la presencia de G. cubensis y distinguirla de otras especies crípticas como Galba truncatula (Müller, 1774) y Galba shirazensis (Küster, 1862) en la región noreste y sur de República Dominicana. Se capturaron moluscos compatibles fenotípicamente con G. cubensis y se tomaron mediciones conquiológicas de los mismos. Además, se realizaron análisis de ADN mediante PCR convencional con primers específicos para las tres especies sospechadas, con ejemplares fijados en alcohol al 80 \%. Se obtuvo amplificación de una región del ADN mitocondrial de la especie $G$. cubensis, únicamente. El uso de la técnica permitió confirmar que $G$. cubensis es, a la fecha, la única especie de limneido involucrada en la transmisión de la fascioliasis en República Dominicana.

Palabras clave: moluscos dulceacuícolas, limneido, hospedero intermediario, fascioliasis, Antillas Mayores.

\section{ABSTRACT}

Among the freshwater gastropods, many of the species of the family Lymnaeidae are involved in the transmission of the parasite trematode Fasciola hepatica (Linnaeus, 1758) worldwide. Galba cubensis (Pfeiffer, 1839) has been reported as a vector in South America and other areas of the Neotropics. The use of molecular techniques has been used by different groups of researchers to discriminate between species with conquiological similarity, such as those of the Galba/Fossaria group, clarifying epidemiological aspects of crucial importance in the study and control of fasciolosis. The objective of this research was to reconfirm with molecular techniques the presence of $G$. cubensis and distinguish it from other cryptic species such as Galba truncatula (Müller, 1774) and Galba shirazensis (Küster, 1862) in the northeastern 
and southern region of the Dominican Republic. Mollusks phenotypically compatible with G. cubensis were captured and conquiological measurements were taken. In addition, conventional PCR DNA analysis was carried out with specific primers for the three suspected species, with specimens fixed in $80 \%$ alcohol. Amplification was obtained from a mitochondrial DNA region of the species $G$. cubensis only. The use of the technique confirmed that G. cubensis is, to date, the only lime species involved in the transmission of fasciolosis in the Dominican Republic.

Keywords: freshwater mollusks, limneid, intermediate host, fasciolosis, Greater Antilles.

\section{INTRODUCCIÓN}

Los moluscos fluviales de la familia Lymnaeidae desempeñan un papel preponderante en la transmisión de la fascioliasis, trematodiasis emergente y reemergente a nivel mundial, producida por los distomas Fasciola hepatica (Linnaeus, 1758) en América y Europa y Fasciola gigantica (Cobbold, 1855) en África y Asia. Estos trematodos producen severos daños hepáticos afectando a diversas especies de animales herbívoros, tanto silvestres como domésticos, y al ser humano (Malek, 1985; Mas-Coma et al., 2008, 2009a; Vázquez et al., 2014, 2018). Tanto F. hepatica como sus hospederos intermediarios, que son moluscos dulceacuícolas, presentan una elevada potencialidad de propagación, debido a la capacidad que poseen de establecerse en áreas con diferentes grados de humedad y elevado gradiente altitudinal (Bargues et al., 2007).

En cuanto a los caracoles, la similitud fenotípica entre especies de limneidos del grupo Galba/Fossaria impide la correcta identificación conquiológica de especímenes, lo que ha generado dudas sobre la verdadera identidad de especies endémicas en países de Sudamérica, América Central e islas del Caribe (Mas-Coma et al., 2001; Vázquez et al., 2016, 2018). Estos moluscos vectores, así como el propio parásito, han desarrollado elevada plasticidad territorial (Correa et al., 2011), factor que, unido a la capacidad de autofecundación dado el hermafroditismo de las especies, les facilita adaptarse y colonizar diferentes hábitats ocupados por otras especies del mismo género u otros (Armúa-Fernández et al., 2016). Esto posibilita su incorporación en la cadena evolutiva del parásito, creando nuevos focos de transmisión de la enfermedad y generando confusión y conflictos taxonómicos entre malacólogos.

En este sentido, desde hace algunas décadas el uso de las técnicas moleculares ha evidenciado la complejidad en las relaciones entre el parásito y sus hospederos, aspecto muy importante en la comprensión de la dinámica de la infección y la creación de nuevos focos de transmisión (Hawley y Altizer, 2011). Schniebs et al., (2018) señalan que, debido al elevado número de notificaciones, tanto de moluscos presentes en nuevas áreas antes insospechadas como de humanos infectados con fascioliasis, es necesario la revisión constante de la situación epidemiológica tradicional de la parasitosis (Mas-Coma et al., 1999a). Esta enfermedad ha dejado de considerarse como una simple zoonosis, sino como una enfermedad transmitida y mantenida por el propio ser humano (Mas-Coma et al., 1999b, 2009b; Mas-Coma, 2005).

El desarrollo de las técnicas moleculares y su aplicación en el diagnóstico fiable de moluscos dulceacuícolas y parásitos ha permitido conocer la distribución geográfica, rango de hospederos, posición filogenética, interacción parásito-hospedero y ciclo de vida de especies de las que hasta ahora se carecía de información (Armúa-Fernández et al., 2016). Por tal motivo, el objetivo de la presente investigación fue la reconfirmación de la especie Galba cubensis (Pfeiffer, 1839), anteriormente Lymnaea cubensis, principal transmisor de F. hepatica en las Américas e islas del Caribe (Álvarez-Vicioso y Cordero de Castellanos, 1977; Gómez et al., 1986; Vázquez et al., 2018) mediante el análisis morfométrico de sus conchas y por técnicas moleculares, a partir de especímenes de limneidos capturados en diferentes localizaciones de República Dominicana. 


\section{MATERIALES Y MÉTODOS}

Área y periodo de estudio. Las muestras de limneidos fueron recogidas en áreas con antecedentes de transmisión de F. hepatica, concretamente en tres localizaciones del noroeste del país: 1) Guayubín, Hatillo de Palma (D.M.), provincia de Monte Cristi; 2) Laguna Salada, Loma de Guayacanes (D.M.), provincia de Valverde; y 3) Laguna Salada, Cruce de Guayacanes (D.M.), provincia de Valverde. Además, también se realizó un muestreo en la región sureste, concretamente en: 4) La Victoria (D.M.), Santo Domingo Norte, provincia de Santo Domingo (Fig. 1). Los muestreos se realizaron durante el mes de octubre de 2017.

Captura y manejo de los moluscos. Los moluscos con fenotipo de limneidos fueron recogidos directamente en la naturaleza, del cieno de hábitats naturales como acequias a orillas de arrozales, potreros con lodazales y gramales, con pinzas de presión suave para no maltratar a los especímenes. Después, se colocaron envueltos en papel toalla húmedos en pequeños contenedores plásticos para ser trasladados vivos y sin deterioro de las conchas hasta el Laboratorio de Fasciola hepatica del Instituto Tecnológico de Santo Domingo (INTEC).

Una vez en el laboratorio, una submuestra de dos moluscos vivos, de cada área, fue fijada en alcohol etílico al $80 \%$, en tubos Eppendorf de $1.5 \mathrm{ml}$, y guardados en el congelador a $-20{ }^{\circ} \mathrm{C}$ para fines de conservación y posterior extracción de ADN. El resto de especímenes vivos se acomodaron en placas con alimento, para adaptarlos al laboratorio y realizar otras investigaciones.

Análisis morfométrico de las conchas. Con los moluscos que habían muerto en el traslado o durante el periodo de adaptación al laboratorio, se realizó un análisis descriptivo de las conchas, cuyas variables de medición fueron obtenidas en el plano empírico, comparando medidas de tendencia central y medidas de dispersión. Para ello, se usaron las conchas previamente lavadas con agua varias veces hasta desprendimiento espontaneo del cuerpo, y luego fueron introducidas en hipoclorito de sodio puro $(\mathrm{NaClO})$, también conocido como cloro comercial. Una vez limpias, se secaron sobre papel toalla y se guardaron en placas Petri hasta el momento de realizar las mediciones.

Cuidando que estuviesen representados debidamente todos los tamaños, se retiraron treinta conchillas (10 grandes, 10 medianas y 10 pequeñas, con longitudes de concha de entre 5-17 mm, 4-15 $\mathrm{mm}$ y 3-8 $\mathrm{mm}$, respectivamente, de acuerdo a la especie) de cada área de estudio, sumando un total de 120 conchillas. Las medidas fueron realizadas con ayuda de un estereomicroscopio Optika ${ }^{\circledR}$ debidamente calibrado, y con la ayuda del programa de edición y tratamiento de imágenes Optika IS view. Las variables medidas fueron: longitud y ancho de la concha; longitud y ancho de la abertura; y longitud de la última vuelta. Además, se determinó el número de vueltas de la espira, posición y forma de la abertura. Como datos adicionales se observó si las conchas eran perforadas o imperforadas, tipo de sutura, forma de la abertura, así como el color natural de la concha.

Para determinar la longitud de la concha, siempre con la abertura de frente al observador y la espira hacia arriba, se trazó una línea recta imaginaria sobre la columela, que es la columna interna donde se enrolla el cuerpo blando del molusco, desde el ápice o vuelta embrionaria hasta el peristoma o labio basal, es decir, hasta el borde inferior de la última vuelta del cuerpo. El ancho de la concha se midió trazando una línea recta imaginaria desde la parte más ancha del lado izquierdo de la última vuelta del cuerpo hasta la más ancha del lado derecho. La longitud de la abertura se midió de igual manera que la longitud total del cuerpo, con la salvedad de que solo se incluyó la porción distal a columella, que se encuentra abierta. Todas las medidas de los cuatro lotes de moluscos fueron promediadas y realizadas por una misma persona, para evitar discrepancias debidas al manipulador. 
Preparación de las muestras para las técnicas moleculares. Dos moluscos, de cada una de las cuatro áreas muestreadas y fijados en alcohol $80 \%$, fueron colocados sobre un portaobjetos $\mathrm{y}$, con la ayuda de otro, se ejerció presión hasta romper las conchas. Con la ayuda de pinzas y bajo el estereomicroscopio, se retiraron todos los restos de concha, para después separar el pie musculoso o masa cefalopodal del resto de vísceras, realizando un corte transversal con bisturí. Este material se colocó nuevamente en alcohol etílico a $80 \%$ y se guardó en el congelador a $-20{ }^{\circ} \mathrm{C}$ hasta el momento de la extracción del ADN.

Extracción de ADN. Para el procedimiento de extracción de ADN se utilizó el protocolo de Doyle \& Doyle (1987); para la aplicación de dicho procedimiento, se prepararon las siguientes soluciones: buffer de lisis $(50 \mathrm{mM}$ de Tris $\mathrm{HCl}, 50 \mathrm{mM}$ EDTA pH $8.1 \%$ de SDS y $50 \mathrm{mM}$ de $\mathrm{NaCl})$. Proteinasa $\mathrm{K}(10 \mathrm{mg} / \mathrm{ml}), \mathrm{NaCl}(5 \mathrm{M}), \mathrm{TE} 1 \mathrm{X} \mathrm{pH} 8$ (autoclavado). Isopropanol absolutos a $-20^{\circ} \mathrm{C}$.

El tejido de las muestras se maceró en un tubo de $1.5 \mathrm{ml}$ con un pistilo y agregando nitrógeno líquido tres veces por cada muestra, con la finalidad de evitar la degradación del ADN por las DNasas. Posteriormente, el ADN extraído se secó en estufa a $37^{\circ} \mathrm{C}$ durante 1 hora, con el propósito de extraer soluciones que interfieran la PCR. Inmediatamente se resuspendió en 30 ul 1xTE. Luego de la extracción, la presencia de ADN se comprobó mediante electroforesis submarina en gel de agarosa al $1 \%$, con solución amortiguadora de Tris-borato-EDTA (TBE) teñida con bromuro de etidio $(\mathrm{BrEt})(0.5 \mathrm{mg} / \mathrm{L})$, esto último para la visualización de los fragmentos de ADN amplificados por PCR. Posteriormente se colocó un Step ladder de $50 \mathrm{pb}$ en los extremos.

La corrida de los ácidos nucleicos se realizó a una corriente de $85 \mathrm{~V}$, durante $15 \mathrm{~m}$. Para visualizar el ADN se utilizó un transiluminador de luz UV, con una longitud de onda de $260 \mathrm{~nm}$. Las muestras restantes de $\mathrm{ADN}$ fueron refrigeradas a $-20{ }^{\circ} \mathrm{C}$, para su posterior PCR.

Para el cóctel de PCR se agregó $1 \mu 1$ de cada primer forward y reverse, Mezcla Master Mix 2X, "Go Taq Hot Start Green"; $13 \mu \mathrm{l} .12 \mu \mathrm{l}$ de $\mathrm{H}_{2} \mathrm{O}$ "nuclease free water", $6 \mu 1$ de ADN y el total de reacción fue de: $33 \mu \mathrm{l}$ volumen final. Para el diseño de primers, se obtuvieron secuencias parciales (Tabla I) de genes de ADN mitocondrial de la subunidad citocromo oxidasa I (COI) tanto de F. hepatica como de G. cubensis, Galba truncatula (Müller, 1774) y Galba schirazensis (Küster, 1862).

La fuente de las secuencias fue del NCBI Genbank Data Base (Tabla II). El acceso KT461812 Versión KT461812.1. Los oligonucleótidos se diseñaron con el programa primer 3. Versión (0.4). La elaboración se hizo en Bioanalytical Instruments, Inc. San Juan, Puerto Rico. El proceso de amplificación fue llevado a cabo en un termociclador "Thermal Cycler PCRSG- 500", bajo las siguientes condiciones de amplificación: temperatura $95{ }^{\circ} \mathrm{C}$ por $3 \min \left(95^{\circ} \mathrm{C}\right.$ por $30 \mathrm{~s}, 55^{\circ} \mathrm{C}$ por $1 \mathrm{~min}, 72{ }^{\circ} \mathrm{C}$ por $1.30 \mathrm{~min}, 30$ ciclos); $72{ }^{\circ} \mathrm{C}$ por $10 \mathrm{~min}$. 

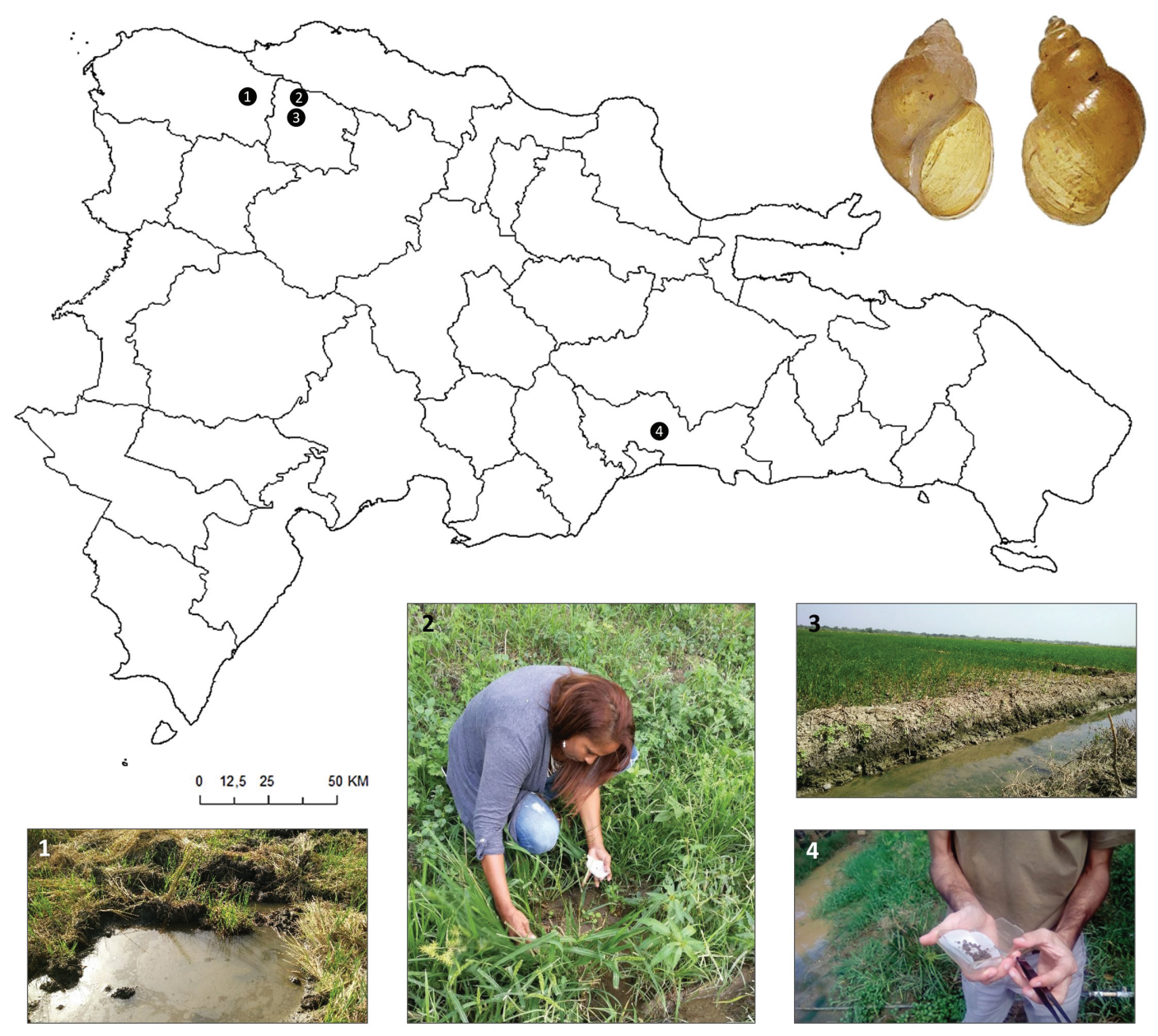

\begin{tabular}{|c|c|c|c|c|c|c|}
\hline & Provincia & Municipio & Latitud & Longitud & Altitud (m s.n.m.) & Tipo de hábitat \\
\hline 1 & Monte Cristi & Guayubín & $19^{\circ} 42^{\prime} 34.00^{\prime \prime} \mathrm{N}$ & $71^{\circ} 12^{\prime} 57.00 " \mathrm{O}$ & 139 & $\begin{array}{l}\text { Montículos de tierra } \\
\text { a orillas de arrozal }\end{array}$ \\
\hline 2 & Valverde & Laguna Salada & $19^{\circ} 42^{\prime} 57.67^{\prime \prime} \mathrm{N}$ & $71^{\circ} 3^{\prime} 35.93^{\prime \prime O} \mathrm{O}$ & 493 & $\begin{array}{c}\text { Zanja frente al Hato } \\
\text { Chávez, Loma de } \\
\text { Guayacanes }\end{array}$ \\
\hline 3 & Valverde & Laguna Salada & $19^{\circ} 42^{\prime} 5.89^{\prime \prime} \mathrm{N}$ & $71^{\circ} 3^{\prime} 35.95^{\prime \prime} \mathrm{O}$ & 310 & $\begin{array}{c}\text { Acequia a orillas de } \\
\text { arrozal, Cruce de } \\
\text { Guayacanes }\end{array}$ \\
\hline 4 & Santo Domingo & $\begin{array}{l}\text { Santo Domingo } \\
\text { Norte }\end{array}$ & $18^{\circ} 34^{\prime} 22.80^{\prime \prime} \mathrm{N}$ & $69^{\circ} 50^{\prime} 52.80^{\prime \prime} \mathrm{O}$ & 10 & $\begin{array}{c}\text { Cenagal de Hato de } \\
\text { Molina }\end{array}$ \\
\hline
\end{tabular}

Figura 1. Área de estudio y localizaciones con presencia de Galba cubensis. 
Tabla I. Secuencia parcial del gen mitocondrial para Galba cubensis, G. schirazensis, G. truncatula y F. hepatica

\begin{tabular}{|c|c|}
\hline Especie & Secuencia parcial \\
\hline G. cubensis & $\begin{array}{l}\text { CAAAATCATAAAGATATTGGTACTTTATATATAATCTTTGGAATCTGATGTGGGTTAGTAGGGACTGGAT } \\
\text { TGTCTTTATTAATTCGACTTGAATTAGGAACATCTACTGTTCTAATTGATGAGCATTTTTATAATGTTAT } \\
\text { TGTTACGGCACATGCATTTGTTATAATTTTTTTTATAGTTATACCTATAATAATTGGTGGGTTTGGAAAC } \\
\text { TGAATAGTACCTTTATTAATTGGTGCACCAGATATAAGATTTCCTCGAATAAATAATATAAGATTTTGAT } \\
\text { TACTACCACCGTCTTTTATTCTTCTTTTATGTTCAAGAATAGTTGAAGGTGGTGTTGGTACGGGATGAAC } \\
\text { AGTTTATCCTCCTTTAAGTGGCCCGATTGCTCATGGAGGGTCATCAGTTGATCTTGCTATTTTTTCATTA } \\
\text { CATTTAGCTGGTCTTTCGAGAATTTTAGGTGCTATTAATTTTATTACTACTATTTTTAACATACGTTCTC } \\
\text { CTGGAATTACTTTGGAACGAATGAGATTATTTGTATGGTCTGTTCTAGTTACTGCTTTCTTATTACTTTT } \\
\text { ATCGTTACCTGTATTAGCTGGAGCGATTACTATACTTTTAACAGATCGAAATTTTAACACTTCTTTTTTT } \\
\text { GATCCAGCTGGAGGTGGTGATCCT }\end{array}$ \\
\hline G. schirazensis & $\begin{array}{l}\text { CATAAAGATATTGGTACTTTATATATAATTTTTGGGGTTTGATGTGGTTTAGTTGGTACCGGTTTATCCT } \\
\text { TACTAATTCGATTAGAACTAGGTACATCTACAGTTTTAATTGATGAACATTTCTATAATGTAATTGTTAC } \\
\text { AGCACATGCTTTTGTTATAATTTTTTTTATAGTTATACCAATAATAATTGGAGGGTTTGGAAATTGAATA } \\
\text { GTACCTTTATTGATTGGTGCTCCTGATATAAGATTCCCACGAATGAATAATATAAGATTTTGATTACTCC } \\
\text { CTCCATCTTTTATTCTTCTTTTATGTTCAAGTATAGTTGAAGGAGGAGTTGGTACAGGTTGAACCGTTTA } \\
\text { CCCTCCTTTAAGAGGACCTATTGCTCATGGTGGATCCTCAGTTGATTTAGCTATTTTTTCTTTACATTTA } \\
\text { GCTGGTCTCTCTAGAATTTTAGGTGCTATTAATTTTATTACTACGATTTTTAATATACGCTCTCCAGGTA } \\
\text { TTACTTTAGAACGAATAAGATTATTTGTATGATCTGTATTAGTTACAGCTTTTTTATTACTTTTATCTTT } \\
\text { ACCTGTATTAGCTGGTGCTATTACTATGCTTCTTACAGATCGAAATTTTAATACTTCTTTTTTTGATCCA } \\
\text { GCTGGTGGAGGTGACCCAATTT }\end{array}$ \\
\hline G. truncatula & $\begin{array}{l}\text { GATATTGGTACTTTATATATAATTTTTGGAATTTGATGTGGATTAATTGGAACTGGTTTATCTTTATTAA } \\
\text { TTCGATTGGAGTTGGGAACATCTACGGTTTTAATTGATGAACATTTCTATAATGTAATTGTTACAGCACA } \\
\text { TGCATTTGTTATAATTTTTTTATAGTAATACCTATAATAATTGGTGGATTTGGTAATTGAATAGTGCCT } \\
\text { TTATTAATTGGTGCTCCTGATATAAGATTTCCACGAATAAATAATATAAGGTTTTGATTACTTCCTCCAT } \\
\text { CTTTTATTCTTCTTTTATGTTCAAGCATAGTTGAAGGGGGAGTGGGTACAGGTTGAACAGTTTACCCACC } \\
\text { TTTAAGCGGACCTATTGCCCATGGCGGGTCTTCAGTTGATTTAGCTATTTTTCTCTACATTTAGCAGGT } \\
\text { CTTTCTAGTATTTTAGGTGCTATTAATTTTATTACTACAATTTTCAATATACGATCGCCCGGAATTACTT } \\
\text { TAGAACGAATAAGGTTATTTGTGTGATCTGTTTTAGTTACTGCTTTTCTTTTACTTTTATCTTTACCTGT } \\
\text { ATTAGCTGGAGCAATTACTATGCTTTTAACAGATCGAAACTTCAATACCTCTTTTTTTGATCCAGCCGGG } \\
\text { GGTGGTGATCCTATTTTATATCAACATTTATTTTGATTTTTT }\end{array}$ \\
\hline F. hepatica & $\begin{array}{l}\text { TGTTGTTGAAAGCTTTTTATTTGGGTTTGAGTAGGTTTTTTGCTTTTGTTATAATTATGG } \\
\text { TTTTTGTTGCGTTTTTTATACTTGGTGAGCGTAAGGTGTTGGGTTATATGCAGATTCGTA } \\
\text { AGGGGCCTAATAAGGTTGGTTTGTGAGGTTTGTTGCAGAGGTTTGCGGATTTAATGAAGT } \\
\text { TAGTTATAAAGTTTAAGTTTGTGTTTTTCAGAATCGTAGTTGGTTGTCTTGGTGGGGTG } \\
\text { TTTATTTGTTGGTTTTGTTGGCTTGTGGCTATTGTGTGTTGTTTTTTTTAGGTTTGGTG } \\
\text { GTGTTAGTAGTGTTAAATTTATGTTGTGGTTTTTAGTGGTTACTAGTATGACTGGTTATA } \\
\text { GGTTGTTAAGTGTTGGTTGGGGTTGTTATAATAAGTTTGCTTTGGTTAGCTGTGTTCGTT } \\
\text { CTGCTTTTGGGTCTGTTAGGTTTGAGGCTTGTTTTATGTGTATTGTTGTTTTGTTTGCAT } \\
\text { TGGTTTGGGGGAGTTATGGTGTTTCTTGTTTGTTTGGTGAATTTGGTGGTATGTG }\end{array}$ \\
\hline
\end{tabular}

Tabla II. Cebadores empleados en la amplificación de moluscos de la especie Galba cubensis

\begin{tabular}{|c|c|c|c|}
\hline Cebador & Secuencia & $\begin{array}{l}\text { Fragmento } \\
\text { amplificado }\end{array}$ & Región de ADN \\
\hline Galba cubensis 1 . Left & attggtgggtttggaaactg & $123-200 \mathrm{pb}$ & Gen mitocondrial \\
\hline Galba cubensis 1. Right & actgatgaccetccatgagc & & \\
\hline
\end{tabular}

\section{RESULTADOS}

Morfometría de la concha. Cónica elongada, de crecimiento lento, cada vuelta es de mayor tamaño que la precedente, con una vuelta del cuerpo desarrollada y crecimiento hacia la derecha. No posee opérculo ni ombligo, aunque algunos especímenes exhiben un pseudombligo. La concha es generalmente frágil, dextrógira, con la abertura de forma semilunar, color cuerno amarillento, marrón antes del lavado con hipoclorito sódico. 
En cuanto a sus medidas, su largo oscila entre 5.2 a $8 \mathrm{~mm}$, y su ancho entre 2 y $3.2 \mathrm{~mm}$; abertura de $2.6 \mathrm{~mm}$ de largo y 1.4 de ancho; longitud de la última vuelta de $4.1 \mathrm{~mm}$. Con frecuencia se observa elevada variabilidad en cuanto al número de vueltas o giros, y posee suturas dentadas, como apunta Malek (1985) y Gómez et al., (1986) (Tabla III).

Análisis de PCR. La electroforesis de los productos de las PCR amplificados con los cebadores o primers específicos de $G$. cubensis revela una banda de 123 a $200 \mathrm{pb}$. Como se puede observar en la Figura 3, hubo amplificación en los pocillos 1, 2, 3, 17, 18, 21, 38 y 42, correspondiente a genes parciales mitocondriales para CO1. No se evidenció infección con F. hepatica.

Tabla III. Promedio morfométrico de 120 moluscos de la especie Galba cubensis recogidos de cuatro áreas con transmisión de F. hepatica en República Dominicana.

\begin{tabular}{cccccccc}
\hline Medida & $\begin{array}{c}\text { Longitud } \\
\text { de concha } \\
(\mathbf{m m})\end{array}$ & $\begin{array}{c}\text { Ancho de } \\
\mathbf{c o n c h a} \\
\mathbf{( m m})\end{array}$ & $\begin{array}{c}\text { Altura de } \\
\text { abertura } \\
\mathbf{( m m})\end{array}$ & $\begin{array}{c}\text { Ancho de } \\
\text { abertura } \\
\mathbf{( m m )}\end{array}$ & $\begin{array}{c}\text { Ángulo } \\
\text { de concha } \\
\text { (grados) }\end{array}$ & $\begin{array}{c}\text { No de } \\
\text { vueltas }\end{array}$ & $\begin{array}{c}\text { Longitud } \\
\text { de última } \\
\text { vuelta } \\
(\mathbf{m m})\end{array}$ \\
Media & 5.2 & 3.2 & 2.6 & 1.4 & 42.1 & 4.1 & 4.1 \\
$\begin{array}{c}\text { Desviación } \\
\text { estándar }\end{array}$ & 1.47 & 0.85 & 0.81 & 0.57 & 10.20 & 0.58 & 1.17 \\
$\begin{array}{c}\text { Valor } \\
\text { máximo }\end{array}$ & 8 & 5 & 4 & 3 & 59 & 5 & 6 \\
$\begin{array}{c}\text { Valor } \\
\text { mínimo }\end{array}$ & 3 & 2 & 1 & 1 & 23 & 3 & 2 \\
\hline
\end{tabular}

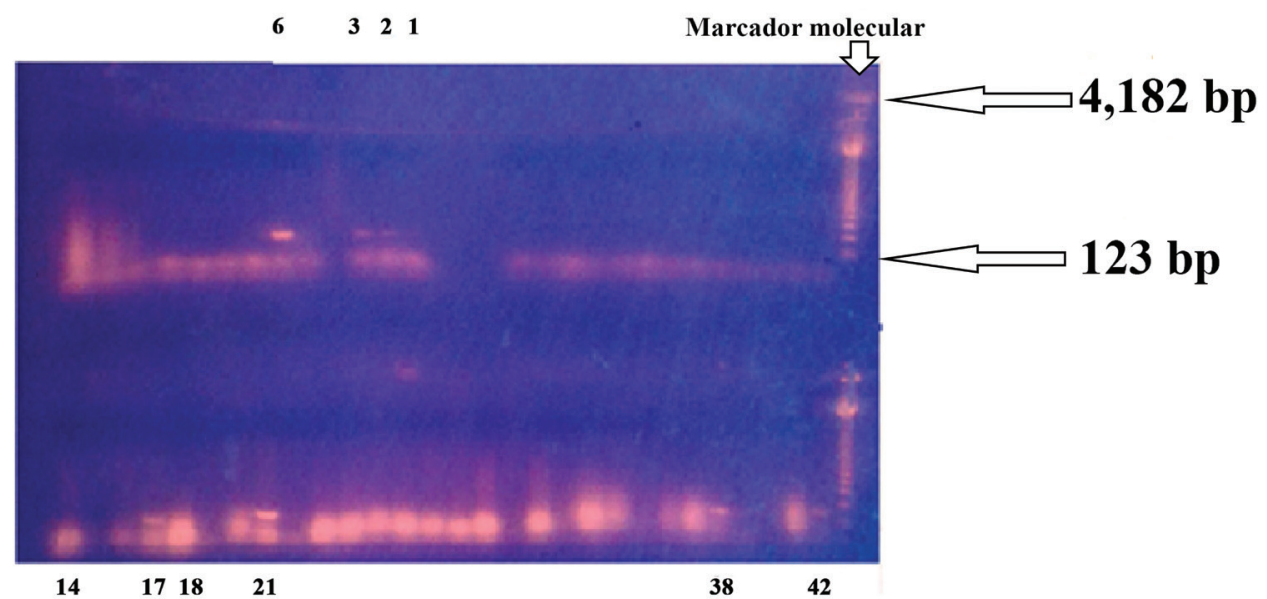

Figura 2. Amplificación de ADN de cuatro morfotipos de muestras de moluscos de la especie Galba cubensis. Visualización en un gel de agarosa al $1 \%$ en Tris Borato EDTA. Los pocillos 1, 2, 3, 17, 18, 21, 38 y 42 resultaron positivos para Galba cubensis, el DNA Ladder de 123 bp en los extremos. 

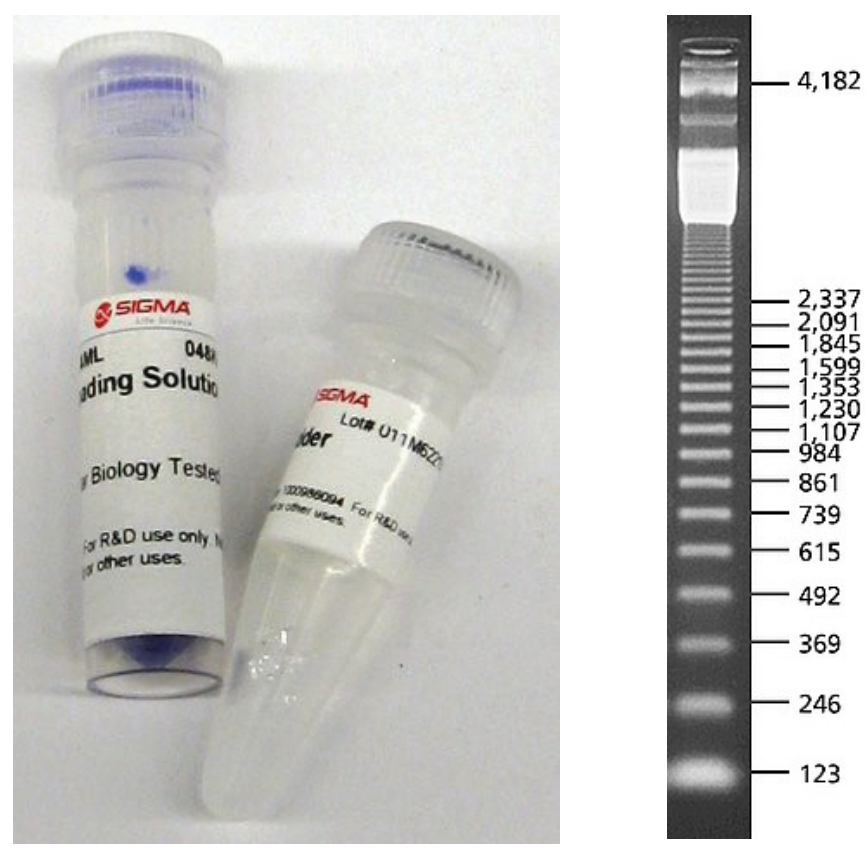

Figura 3. Marcador molecular de 123 bp de Sigma Aldrich, utilizado en la electroforesis en gel de agarosa al $1 \%$ en Tris Borato EDTA.

\section{DISCUSIÓN}

Tradicionalmente, la identificación de los moluscos de importancia médica y veterinaria, como algunas especies de la familia Lymnaeidae, se ha basado en el estudio anatomo-morfológico de ciertas partes de interés: estudio morfométrico de las conchas, disección anatómica y estudio morfométrico de los genitales masculinos y femeninos, especialmente del prepucio, y morfología de los dientes de la rádula. Estos procedimientos eran considerados como los métodos de referencia en la identificación de los moluscos. Sin embargo, se empleaba mucho tiempo en estos menesteres, los cuales eran privilegio tan solo de unos pocos científicos (Paraense, 1983; Malek, 1985; Gómez et al., 1986; Pointier et al., 2006, 2009).

En el caso de G. cubensis, es difícil realizar la distinción con el resto de especies crípticas transmisoras de F. hepatica mediante técnicas meramente conquiológicas. Esto es de gran importancia epidemiológica ya que es un molusco ampliamente identificado como vector del trematodo en el Neotrópico (Bargues et al., 2007; Vázquez et al., 2018); sin embargo, Álvarez-Vicioso y Cordero de Castellanos (1977) y Gómez et al. (1986) incriminan a esta especie como responsable de dispersar la fascioliasis bovina en República Dominicana, mucho antes de la implementación de las técnicas moleculares.

Con los resultados de las mediciones obtenidas de las conchas de G. cubensis en esta investigación, se desprenden informaciones malacológicas precisas que, comparadas con otras poblaciones del mismo molusco u otras especies, facilitaría la colecta de muestras para el diagnóstico ocular a personal no especialista. En República Dominicana, un buen ejemplo de ello serían los promotores del control de la malaria en sus búsquedas de larvas de mosquitos anofelinos en ecosistemas hídricos donde, además, se multiplican estas especies de limneidos. Asimismo, esta información puede facilitar la selección previa de las muestras, para luego confirmar la identidad con técnicas moleculares. Sin embargo, con el surgimiento de las 
técnicas moleculares se ha abierto un amplio campo de estudio con resultados fiables y validables en un $100 \%$ de los casos. Estas nuevas técnicas nos permiten estudiar, además, la relación parásito-hospedero, lo cual es esencial en la comprensión de la dinámica de infección y transmisión (Hawley \& Altizer, 2011).

Han sido varias las especies de limneidos cuya diferenciación se ha realizado gracias a estas nuevas técnicas, incluyendo nuevas especies que se confundían con las señaladas como hospederos intermediarios en el pasado. Armúa-Fernández et al. (2016) reconocieron por primera vez a Galba neotropica como nueva especie en Uruguay, mediante caracterización molecular, e identificaron tres genes amplificados (ITS2, COI y 16S) utilizando protocolos de PCR previamente descriptos, logrando con esto la determinación de similitud entre especies y su parentesco mediante análisis filogenéticos. De igual manera, Standley et al. (2013) identificaron, con ayuda de técnicas moleculares, a G. neotropica en la Patagonia Argentina, donde solo se conocía la presencia de G. truncatula y Galba viatrix (d'Orbigny, 1835); además, esclarecieron que $G$. truncatula aparecía en lugares a más de 1900 m s.n.m., G. neotropical $G$. viatrix conjuntamente en altitudes medias de 1300 a 1900 m s.n.m., y G. viatrix sola en lugares con altitudes menores a 1300 m s.n.m. En el mismo estudio, el análisis filogenético utilizando dos marcadores mitocondriales reveló que $G$. neotropica y $G$. viatrix están estrechamente relacionadas y, por su enorme parecido morfológico, debe cuestionarse su validez como entidades taxonómicas separadas. Por otro lado, se ha identificado recientemente en Ecuador a $G$. cubensis en sembradíos de arroz, la cual era confundida con $G$. truncatula; esta especie podría ser transmisora de la $F$. hepatica en la región costera de Ecuador (Narváez et al., 2017).

De entre las especies crípticas que componen el grupo Galba/Fossaria, siendo estas G. schirazensis, G. truncatula, G. cubensis, Galba humilis (Say, 1822), Galba viator (d'Orbigny, 1835) y Galba cousini (Jousseaume, 1887), tan solo la última posee diferencias anatómicas y conquiológicas con el resto (Paraense, 1983; Correa et al., 2011). De igual manera, de las tres especies de limneidos presentes en República Dominicana, solo Pseudosuccinea columella (Say, 1817) puede identificarse atendiendo a sus características conquiológicas (De Vargas Castro et al., 2019).

Con el ensayo de PCR multiplex se ha logrado distinguir exitosamente a G. cubensis, tanto de Sudamérica como de las Antillas Mayores. Lounnas et al. (2017) lograron estudiar molecularmente 13 poblaciones de G. cubensis de Cuba, Guadalupe, Martinica, Puerto Rico, Venezuela, Colombia y Ecuador. En siete de estas poblaciones lograron aislar 15 microsatélites en 359 especímenes; esto les permitió evaluar su baja diversidad genética y alta tasa de cruzamiento, también demostraron una relación estrecha entre G. cubensis y G. neotropica. Alda et al. (2018) alcanzaron a distinguir tres especies del grupo Galba/Fossaria transmisoras de F. hepatica, siendo estas G. cubensis, G. schirazensis y G. truncatula, con la técnica molecular PCR multiplex en un solo intento, siendo que cada especie fue identificada por el tamaño de una banda amplificada producida por ADN de cada especie presente en la misma reacción.

Nuestros resultados coinciden con los Alba et al., (2015) en Cuba, Martínez et al. (2015) en República Dominicana y Alda et al. (2018) en Argentina, al utilizar las mismas herramientas moleculares en la identificación de las cuatro poblaciones de G. cubensis distribuidas en el noreste y sur del país. 


\section{CONCLUSIONES Y RECOMENDACIONES}

La técnica molecular utilizada en este estudio para la reconfirmación de la identificación del molusco fluvial $G$. cubensis arrojó resultados precisos y confiables, más si cabe al haberse conjugado con técnicas malacológicas y conquiológicas que, a nuestro juicio, siempre serán de gran valor en la identificación de especímenes con morfologías sugestivas de las especies pertenecientes a esta familia de notorio interés médico-veterinario.

Galba cubensis es, aparentemente, la especie más abundante de Lymnaeidae en el noreste de República Dominicana, además de en Hato Molina, La Victoria, Santo Domingo Norte. Como consecuencia de no haber sido identificada otra especie de Lymnaeidae y existir constantemente transmisión de $F$. hepatica en las áreas estudiadas, podríamos inferir que G. cubensis es, hasta la fecha, la única especie de limneido involucrado en la transmisión de la fascioliasis en estas zonas del país.

\section{AGRADECIMIENTOS}

El presente trabajo se deriva del proyecto "Transmisión de Fasciola hepatica (Trematoda: Digenea), por sus moluscos hospederos intermediarios, (Gastropoda: Lymnaeidae), en el Caribe Insular", Fondo Nacional de Innovación y Desarrollo Científico y Tecnológico (FONDOCyT), del Ministerio de Educación Superior, Ciencia y Tecnología (MESCyT), República Dominicana. Asimismo, deseamos dedicar esta investigación a la memoria de los Profesores José de JesúsÁlvarez Vicioso, José Gómez Pérez y Emile A. Malek, pioneros de la ciencia de la Malacología Médica en República Dominicana.

\section{LITERATURA CITADA}

Alba, A., A. A. Vázquez, H. Hernández, J. Sánchez, R. Marcet, M. Figueredo, J. Sarracent y J. Fraga. 2015. A multiplex PCR for the detection of Fasciola hepatica in the intermediate snail host Galba cubensis. Veterinary Parasitology, 211 (3-4): 195-200.

Alda, P., M. Lounnas, A. A. Vázquez, R. Ayaqui, M. Calvopiña, M. Celi-Erazo, R. T. Dillon, Jr., P. Jarne, E. S. Loker, F. C. Muñiz Pareja, J. Muzzio-Aroca, A. O. Nárvaez, O. Noya, L. M. Robles, R. Rodríguez-Hidalgo, N. Uribe, P. David, J. P. Pointier y S. Hurtrez-Boussès. 2018. A new multiplex PCR assay to distinguish among three cryptic Galba species, intermediate hosts of Fasciola hepatica. Veterinary Parasitology, 251: 101-105.

Álvarez-Vicioso, J. M. y E. T. Cordero de Castellanos. 1977. Faciolasis bovina en la República Dominicana. Ed. Universidad Autónoma de Santo Domingo, Santo Domingo: República Dominicana, 189 pp.

Armúa-Fernández M. T., O. Castro, O. Correa, L. Carvalho, A. Mangold, J. Sanchís y J. M. Venzal. 2016. First molecular characterization of Galba neotropica in Uruguay. Revista FAVE - Sección Ciencias Veterinarias, 15: 9-13

Bargues, M. D., P. Artigas, Y. Mera, R. L. Sierra, J. P. Pointier y S. Mas-Coma. 2007. Characterisation of Lymnaea cubensis, L. viatrix and L. neotropica n. sp., the main vectors Fasciola hepatica in Latin America, by analysis of their ribosomal and mitochondrial DNA. Annals of Tropical Medicine and Parasitology, 101 (7): 621-641. 
Correa, A. C., J. S. Escobar, O. Noya, L. E. Velásquez, C. González-Ramírez, S. HurtrezBoussès y J. P. Pointier. 2011. Caracterización morfológica y molecular de Lymnaeidae (Gastropoda: Hygrophila) de la región neotropical. Biomédica, 31(sup.3): 201-206.

De Vargas Castro, M., J. Martínez La Hoz, J. Castillo, D. Paulino, P. M. Alarcón-Elbal y A. A. Vázquez. 2019. Potencialidad de Pseudosuccinea columella (Say, 1817) (Molusca: Gastropoda: Lymnaeidae) en la transmisión de la fascioliasis humana en República Dominicana. Ciencia y Salud, 3 (2): 9-16.

Doyle, J. J. y J. L. Doyle. 1987. A rapid DNA isolation procedure for small quantities of fresh leaf tissue. Phytochemical Bulletin, 19: 11-15.

Gómez, J. D, M. Vargas y E. A. Malek. 1986. Freshwater molluks of the Dominican Republic. The Nautillus, 100 (4): 130-134.

Hawley, D. M. y S. M. Altizer. 2011. Disease ecology meets ecological immunology: understanding the links between organismal immunity and infection dynamics in natural populations. Functional Ecology, 25: 48-60.

Lounnas, M., A. A. Vázquez, P. Alda, K. Sartori, J. P. Pointier, P. David y S. Hurtrez-Boussès, 2017. Isolation, characterization and population-genetic analysis of microsatellite loci in the freshwater snail Galba cubensis (Lymnaeidae). Journal of Molluscan Studies, 83 (1): 63-68.

Malek, E. A. 1985. Snail Hosts of Schistosomiasis and other Snail-Transmitted Diseases in Tropical America: a Manual. Pan American Health Organization, Washington: USA, $325 \mathrm{pp}$.

Martínez, M. A., P. Artigas, L. Mateo, V. Chagas, S. Adam, S. Mas-Coma y M. D. Bargues. 2015. Identificación de Lymnaea cubensis en zonas endémicas de Fasciolasis en República Dominicana. XIX Congreso de la Sociedad Española de Parasitología: 23, 24 y 25 de julio de 2015; Vitoria-Gasteiz (España). SOCEPA; 2015.

Mas-Coma, S. 2005. Epidemiology of fascioliasis in human endemic areas. Journal of Helminthology, 79 (3): 207-216.

Mas-Coma, M. S., J. G. Esteban y M. D. Bargues. 1999a. Epidemiología de la fascioliasis humana: revisión y propuesta de nueva clasificación. Boletín de la Organización Mundial de la Salud, Recopilación de artículos N¹: 70-76.

Mas-Coma, S., R. Anglés, J. G. Esteban, M. D. Bargues, P. Buchon, M. Franken y W. Strauss. 1999b. The Northern Bolivian Altiplano: A región highly endemic for human fasciolasis. Tropical Medicine and International Health, 4 (6): 454-467.

Mas-Coma, S., I. R. Funatsu y M. D. Bargues. 2001. Fasciola hepatica and lymnaeid snails occurring at very high altitude in South America. Parasitology, 123 Suppl: S115-27.

Mas-Coma, S., M. A. Valero y M. D. Bargues. 2008. Effects of climate change on animal and zoonotic helminthiases. Revue Scientifique et Technique (International Office of Epizootics), 27: 443-457. 
Mas-Coma, S., M. A. Valero y M. D. Bargues. 2009a. Climate change effects on trematodiases, with emphasis on zoonotic fascioliasis and schistosomiasis. Veterinary Parasitology, 163 (4): 246-280.

Mas-Coma, S., M. A. Valero y M. D. Bargues. 2009b. Chapter 2. Fasciola, lymnaeids and human fascioliasis, with a global overview on disease transmission, epidemiology, evolutionary genetics, molecular epidemiology and control. Advances in Parasitology, 69: 41-146.

Narváez, A. O., J. Muzzio Aroca, P. Alda, V. Macías Castro, M. Lounnas, S. Hurtrez-Boussès, O. Noya, L. Martini Robles y J. P. Pointier. 2017. Primer reporte de Galba cubensis (Gastropoda: Lymnaeidae) en el Ecuador, hospedador potencial de Fasciola hepatica en arrozales de la costa ecuatoriana. El Misionero del Agro, 13 (4): 36-47.

Paraense, W. L.1983. Lymnaea columella in northern Brazil. Memórias do Instituto Oswaldo Cruz, 78 (4): 477-482.

Pointier, J. P., N. J. Cazzaniga, C. González-Salas, A. Gutiérrez, J. A. Arenas, M. D. Bargues y S. Mas-Coma. 2006. Anatomical studies of sibling species within neotropical lymnaeids, snail intermediate hosts of fascioliasis. Memórias do Instituto Oswaldo Cruz, 101 (4): 431-435.

Pointier, J. P., O. Noya, B. Alarcón de Noya y A. Théron, 2009. Distribution of Lymnaeidae (Mollusca: Pulmonata) intermediate hosts of Fasciola hepatica in Venezuela. Memórias do Instituto Oswaldo Cruz, 104 (5): 790-796.

Schniebs, K., P. Gloer, S. Quiñonero-Salgado, J. López-Soriano y A. K. Hundsdoerfer. 2018. The first record of Galba cubensis (L. Pfeiffer, 1839) (Mollusca: Gastropoda: Lymnaeidae) from open fields of Europe. Folia Malacologica, 26 (1): 3-15.

Standley, C. J., L. Prepelitchi, S. M. Pietrokovsky, L. Issia, J. R. Stothard y C. WisniveskyColli. 2013. Molecular characterization of cryptic and sympatric lymnaeid species from the Galba/Fossaria group in Mendoza Province, Northern Patagonia, Argentina. Parasites \& Vectors, 6 (1): 304.

Vázquez, A.A, J. Sánchez, J. P. Pointier, A. Théron y S. Hurtrez-Boussès. 2014. Fasciola hepática in Cuba: compatibility of different isolates with two intermediate snail hosts, Galba cubensis and Pseudosuccinea columella. Journal of Helminthology, 88 (4): 434-440.

Vázquez, A. A., M. Lounnas, J. Sánchez, A. Alba, A. Milesi y S. Hurtrez-Boussès. 2016. Genetic and infective diversity of the liver fluke Fasciola hepatica (Trematoda: Digenea) from Cuba. Journal of Helminthology, 90 (6): 719-725.

Vázquez, A. A, P. Alda, M. Lounnas, E. Sabournin, J. P Pointier y S. Hurtrez-Boussés. 2018. Lymnaeidae snails host of Fasciola hepatica and Fasciola gigantica (Trematoda: Digenea): A wordlwide review. CAB Reviews: Perspectives in Agriculture, Veterinary Science, Nutrition and Natural Resources, 13 (062): 1-15.

[Recibido: 19 de agosto, 2019. Aceptado para publicación: 05 de noviembre, 2019] 